\title{
Deterministic and stochastic methods for computing volumetric moduli of convex cones
}

\author{
DANIEL GOURION and ALBERTO SEEGER \\ University of Avignon, Department of Mathematics \\ 33 rue Louis Pasteur, 84000 Avignon, France
}

E-mails: daniel.gourion@univ-avignon.fr / alberto.seeger@univ-avignon.fr

\begin{abstract}
This work concerns the practical computation of the volumetric modulus, also called normalized volume, of a convex cone in a Euclidean space of dimension beyond three. Deterministic and stochastic techniques are considered.
\end{abstract}

Mathematical subject classification: Primary: 28A75; Secondary: 52A20.

Key words: convex cone, volumetric modulus, solid angle, randomness.

\section{Introduction}

Convex cones play a prominent role in many branches of applied mathematics. Throughout this work, $\Xi\left(\mathbb{R}^{n}\right)$ stands for the collection of nontrivial closed convex cones in $\mathbb{R}^{n}$. That a convex cone is nontrivial simply means that it is different from the singleton $\{0\}$ and different from the whole space $\mathbb{R}^{n}$.

Which is the most relevant information concerning the geometric nature of an element $K$ taken from $\Xi\left(\mathbb{R}^{n}\right)$ ? The answer to this question depends very much upon the specific context under consideration. From a measure-theoretic point of view, a natural question is whether or not $K$ occupies a lot of room in the space $\mathbb{R}^{n}$ in comparison with some reference set. The idea of volume of a convex cone is captured by the next definition. Once and for all we assume that $n$ is greater than or equal to three. 
Definition 1.1. Let $K \in \Xi\left(\mathbb{R}^{n}\right)$. The volumetric modulus (or normalized volume) of $K$ is defined as the ratio

$$
\varrho(K)=\frac{\operatorname{vol}_{n}\left(K \cap \mathbb{B}_{n}\right)}{\frac{1}{2} \operatorname{vol}_{n}\left(\mathbb{B}_{n}\right)}
$$

with $\mathbb{B}_{n}$ standing for the closed unit ball in $\mathbb{R}^{n}$.

One truncates $K$ with the ball $\mathbb{B}_{n}$ for obtaining a set with finite volume. Needless to say, " $\mathrm{vol}_{n}$ " refers to the $n$-dimensional volume. The coefficient $1 / 2$ in the denominator of (1) has been introduced on purpose. With such a calibration factor, the ratio $\varrho(K)$ indicates how much room occupies $K$ when compared with a half-space. In contrast to [20], we use a half-space as reference set and not the whole space $\mathbb{R}^{n}$. If one adopts Definition 1.1, then one has the following properties:

$$
\begin{aligned}
& \left\{\varrho(K): K \in \Xi\left(\mathbb{R}^{n}\right)\right\}=[0,1], \\
& \varrho(K)=0 \text { if and only if } K \text { has empty interior, } \\
& \varrho(K)=1 \text { if and only if } K \text { is a half-space. }
\end{aligned}
$$

Instead of focusing on the volume of the convex set $K \cap \mathbb{B}_{n}$, one could perfectly well put the emphasis on the surface that $K$ produces over the unit sphere $\mathbb{S}_{n}$ of $\mathbb{R}^{n}$. Indeed, one has the formula

$$
\varrho(K)=\frac{\operatorname{vol}_{n-1}\left(K \cap \mathbb{S}_{n}\right)}{\frac{1}{2} \operatorname{vol}_{n-1}\left(\mathbb{S}_{n}\right)},
$$

where "areas" are computed with respect to the spherical Lebesgue measure in $\mathbb{S}_{n}$. The numerator of the ratio (3) is sometimes called the solid angle of $K$. The literature on solid angles is quite extensive for the case $n=3$, but there are still important things to be said in higher dimensions.

\section{Preliminaries}

A convex cone is called solid if its interior is nonempty. The property (2) suggests that $\varrho(K)$ can be used as tool for measuring the degree of solidity of $K$. The next proposition shows that the function $\varrho: \Xi\left(\mathbb{R}^{n}\right) \rightarrow \mathbb{R}$ qualifies as index 
of solidity in the sense of [15]. As usual, one defines the distance between two elements $K_{1}, K_{2}$ of the set $\Xi\left(\mathbb{R}^{n}\right)$ by means of the expression

$$
\delta\left(K_{1}, K_{2}\right)=\operatorname{haus}\left(K_{1} \cap \mathbb{B}_{n}, K_{2} \cap \mathbb{B}_{n}\right)
$$

with haus $(\cdot, \cdot)$ standing for the Pompeiu-Hausdorff metric on the collection of all of compact nonempty subsets of $\mathbb{R}^{n}$.

Proposition 2.1. One has:

(a) $\varrho: \Xi\left(\mathbb{R}^{n}\right) \rightarrow \mathbb{R}$ is continuous with respect to the metric $\delta$.

(b) $\varrho: \Xi\left(\mathbb{R}^{n}\right) \rightarrow \mathbb{R}$ is monotonic in the sense that $K_{1} \subset K_{2}$ implies $\varrho\left(K_{1}\right) \leq$ $\varrho\left(K_{2}\right)$.

(c) $\varrho(Q(K))=\varrho(K)$ for any $K \in \Xi\left(\mathbb{R}^{n}\right)$ and any orthogonal matrix $Q$ of order $n$.

Proof. The proof is essentially a matter of exploiting the general properties of the $n$-dimensional Lebesgue measure (monotonicity, orthogonal invariance, etc).

There are only few examples of convex cones for which the volumetric modulus admits an explicit and easily computable formula. The oldest and best known example is recalled below.

Example 2.2. Consider a polyhedral convex cone $K$ in $\mathbb{R}^{3}$ generated by three linearly independent unit vectors $\left\{g^{1}, g^{2}, g^{3}\right\}$. The solid angle of $K$ can be computed by using the equality

$$
\tan \left(\frac{\operatorname{area}\left(K \cap \mathbb{S}_{3}\right)}{2}\right)=\frac{\left|\operatorname{det}\left[g^{1}, g^{2}, g^{3}\right]\right|}{1+\left\langle g^{1}, g^{2}\right\rangle+\left\langle g^{2}, g^{3}\right\rangle+\left\langle g^{1}, g^{3}\right\rangle} .
$$

Hence, the volumetric modulus of $K$ is given by

$$
\varrho(K)=\frac{1}{\pi} \arctan \left(\frac{\left|\operatorname{det}\left[g^{1}, g^{2}, g^{3}\right]\right|}{1+\left\langle g^{1}, g^{2}\right\rangle+\left\langle g^{2}, g^{3}\right\rangle+\left\langle g^{1}, g^{3}\right\rangle}\right) .
$$


If the argument of arctan is negative, then one adds 1 to the right-hand side of (5). The triple product formula (4) is sometimes attributed to van Oosterom and Strackee [21], but, as rightly pointed out by Eriksson [7], such equality appears already in Euler's manuscript "De mensura angulorum solidorum", 1781.

While working in higher dimensional spaces, the computation of a volumetric modulus is usually a cumbersome task, a notable exception being the case of a circular cone

$$
R_{a, \vartheta}=\left\{x \in \mathbb{R}^{n}:\|x\| \cos \vartheta \leq\langle a, x\rangle\right\} .
$$

The parameters $a \in \mathbb{S}_{n}$ and $\vartheta \in[0, \pi / 2]$ stands, respectively, for the revolution axis and the half-aperture angle of the cone. For notational convenience, we introduce the positive constant

$$
\kappa_{n}=\int_{0}^{\pi / 2}(\sin t)^{n-2} d t=\frac{\sqrt{\pi}}{2} \frac{\Gamma\left(\frac{n-1}{2}\right)}{\Gamma\left(\frac{n}{2}\right)}
$$

whose explicit evaluation presents no difficulty. As usual, $\Gamma$ stands for the Euler gamma function.

Proposition 2.3 (cf. [23]). Let $a \in \mathbb{S}_{n}$ and $\vartheta \in[0, \pi / 2]$. Then,

$$
\varrho\left(R_{a, \vartheta}\right)=F_{n}(\vartheta):=\frac{1}{\kappa_{n}} \int_{0}^{\vartheta}(\sin t)^{n-2} d t .
$$

Furthermore,

$$
\frac{\Gamma\left(\frac{n}{2}\right)(\sin \vartheta)^{n-1}}{\Gamma\left(\frac{n+1}{2}\right) \sqrt{\pi} \cos \vartheta}\left[1-\frac{(\tan \vartheta)^{2}}{n}\right] \leq \varrho\left(R_{a, \vartheta}\right) \leq \frac{\Gamma\left(\frac{n}{2}\right)(\sin \vartheta)^{n-1}}{\Gamma\left(\frac{n+1}{2}\right) \sqrt{\pi} \cos \vartheta} .
$$

A short and simple proof of (6) runs as follows. Since $\varrho$ is orthogonally invariant, there is no loss of generality in assuming that $a$ is the first canonical vector of $\mathbb{R}^{n}$. The volume of $R_{a, \vartheta} \cap \mathbb{B}_{n}$ is given by the $n$-fold integral

$$
\begin{array}{r}
2 \int_{0}^{1} \int_{0}^{\vartheta} \int_{0}^{\pi} \ldots \int_{0}^{\pi} r^{n-1}\left(\sin \phi_{1}\right)^{n-2}\left(\sin \phi_{2}\right)^{n-3} \ldots\left(\sin \phi_{n-2}\right) \\
d r d \phi_{1} d \phi_{2} \ldots d \phi_{n-1}
\end{array}
$$


with $r, \phi_{1}, \ldots, \phi_{n-1}$ standing for the usual hyperspherical coordinates. By unfolding the above integral, one gets

$$
\begin{array}{r}
2 \int_{0}^{1} r^{n-1} d r \int_{0}^{\vartheta}\left(\sin \phi_{1}\right)^{n-2} d \phi_{1} \int_{0}^{\pi}\left(\sin \phi_{2}\right)^{n-3} d \phi_{2} \\
\ldots \int_{0}^{\pi} \sin \phi_{n-2} d \phi_{n-2} \int_{0}^{\pi} d \phi_{n-1} .
\end{array}
$$

The half-volume of $\mathbb{B}_{n}$ is computed in the same way, except that integration with respect to $\phi_{1}$ runs now from 0 to $\pi / 2$. By passing to the quotient and removing the terms that cancel out, one ends up with (6).

Proposition 2.3 was obtained and used by Shannon [23] for estimating error probabilities while decoding optimal codes in a Gaussian channel. See [10] for a more updated reference. This is just one of the many areas of application of the concept of volumetric modulus.

Corollary 2.4. The n-dimensional Lorentz cone

$$
L_{n}=\left\{x \in \mathbb{R}^{n}:\left[x_{1}^{2}+\ldots+x_{n-1}^{2}\right]^{1 / 2} \leq x_{n}\right\}
$$

has a volumetric modulus given by

$$
\varrho\left(L_{n}\right)=\frac{1}{\kappa_{n}} \int_{0}^{\pi / 4}(\sin t)^{n-2} d t
$$

Furthermore,

$$
\lim _{n \rightarrow \infty}(\sqrt{2})^{n-3} \sqrt{\pi n} \varrho\left(L_{n}\right)=1 .
$$

Proof. The proof of the corollary is a matter of applying Proposition 2.3 with $\vartheta=\pi / 4$. The asymptotic behavior of $\varrho\left(L_{n}\right)$ follows by combining the sandwich

$$
\frac{\Gamma\left(\frac{n}{2}\right)}{\Gamma\left(\frac{n+1}{2}\right) \sqrt{\pi}}\left(\frac{\sqrt{2}}{2}\right)^{n-2}\left(1-\frac{1}{n}\right) \leq \varrho\left(L_{n}\right) \leq \frac{\Gamma\left(\frac{n}{2}\right)}{\Gamma\left(\frac{n+1}{2}\right) \sqrt{\pi}}\left(\frac{\sqrt{2}}{2}\right)^{n-2}
$$

and Stirling's approximation formula for factorials.

\section{Numerical integration method}

We next address the problem of evaluating the volumetric modulus of a polyhedral convex cone. The following result can be found in [20], although under a slightly different notation. 
Lemma 3.1. Let $K$ be a polyhedral convex cone in $\mathbb{R}^{n}$ generated by $n$ linearly independent unit vectors $\left\{g^{1}, \ldots, g^{n}\right\}$. Then,

$$
\varrho(K)=2 \frac{\sqrt{\operatorname{det} M}}{\pi^{n / 2}} \int_{\mathbb{R}_{+}^{n}} e^{-\langle\xi, M \xi\rangle} d \xi
$$

with $M=\left[\left\langle g^{i}, g^{j}\right\rangle\right]_{i, j=1, \ldots, n}$ standing for the Gramian matrix associated to the generators.

The multiple integral in (7) can be computed explicitly only in rare circumstances. One favorable situation occurs when the generators of the cone are mutually orthogonal.

Corollary 3.2. Let $K$ be a polyhedral convex cone in $\mathbb{R}^{n}$ generated by $n$ mutually orthogonal unit vectors. Then, $\varrho(K)=(1 / 2)^{n-1}$.

Proof. By orthogonality, $M$ is the identity matrix. Hence,

$$
e^{-\langle\xi, M \xi\rangle}=e^{-\xi_{1}^{2}} \ldots e^{-\xi_{n}^{2}},
$$

and (7) can be evaluated by repeated one-dimensional integration.

The following result can be seen as an extension of Corollary 3.2. For a symmetric matrix $M$, one writes

$$
\begin{aligned}
\mu_{\min }(M) & =\min _{\xi \geq 0,\|\xi\|=1}\langle\xi, M \xi\rangle, \\
\mu_{\max }(M) & =\max _{\xi \geq 0,\|\xi\|=1}\langle\xi, M \xi\rangle,
\end{aligned}
$$

where $\xi \geq 0$ indicates that each component of the vector $\xi$ is nonnegative. The above numbers appear once and over again in linear algebra and optimization. For a practical computation of (8) and (9), one can use for instance the pre-activity method of Seeger and Torki [22, Theorem 3].

Proposition 3.3. Let $K$ be a polyhedral convex cone in $\mathbb{R}^{n}$ generated by $n$ linearly independent unit vectors. Let $M$ be the Gramian matrix associated to the generators. Then,

$$
\left(\frac{1}{2}\right)^{n-1} \frac{\sqrt{\operatorname{det} M}}{\left[\mu_{\max }(M)\right]^{n / 2}} \leq \varrho(K) \leq\left(\frac{1}{2}\right)^{n-1} \frac{\sqrt{\operatorname{det} M}}{\left[\mu_{\min }(M)\right]^{n / 2}} .
$$


Proof. From the definition of $\mu_{\min }(M)$ and $\mu_{\max }(M)$, one sees that

$$
\mu_{\min }(M)\|\xi\|^{2} \leq\langle\xi, M \xi\rangle \leq \mu_{\max }(M)\|\xi\|^{2}
$$

for all $\xi \in \mathbb{R}_{+}^{n}$. Lemma 3.1 completes the proof.

If one does not wish to bother computing the numbers $\mu_{\min }(M)$ and $\mu_{\max }(M)$, then one can use the coarser estimates

$$
\left(\frac{1}{2}\right)^{n-1} \frac{\sqrt{\operatorname{det} M}}{\left[\lambda_{\max }(M)\right]^{n / 2}} \leq \varrho(K) \leq\left(\frac{1}{2}\right)^{n-1} \frac{\sqrt{\operatorname{det} M}}{\left[\lambda_{\min }(M)\right]^{n / 2}}
$$

with $\lambda_{\min }(M)$ and $\lambda_{\max }(M)$ denoting, respectively, the smallest and the largest eigenvalue of $M$.

Example 3.4. Let $K$ be the polyhedral convex cone in $\mathbb{R}^{4}$ generated by the linearly independent unit vectors

$$
g^{1}=\frac{1}{2}\left[\begin{array}{c}
1 \\
-1 \\
-1 \\
1
\end{array}\right], g^{2}=\frac{1}{10}\left[\begin{array}{l}
5 \\
1 \\
7 \\
5
\end{array}\right], g^{3}=\frac{1}{7}\left[\begin{array}{c}
-4 \\
4 \\
1 \\
4
\end{array}\right], g^{4}=\frac{1}{11}\left[\begin{array}{c}
-4 \\
-5 \\
8 \\
4
\end{array}\right]
$$

In this example, the associated Gramian matrix

$$
M=\left[\begin{array}{cccc}
1 & 1 / 10 & -5 / 14 & -3 / 22 \\
1 / 10 & 1 & 11 / 70 & 51 / 110 \\
-5 / 14 & 11 / 70 & 1 & 20 / 77 \\
-3 / 22 & 51 / 110 & 20 / 77 & 1
\end{array}\right]
$$

has both positive and negative off-diagonal entries. A matter of computation yields $\operatorname{det} M=0.607185$ and

$$
\begin{cases}\lambda_{\min }(M)=0.475562, & \lambda_{\max }(M)=1.672900, \\ \mu_{\min }(M)=0.642857, & \mu_{\max }(M)=1.607770 .\end{cases}
$$

In view of (11), one has $0.0348 \leq \varrho(K) \leq 0.4307$. By using (10) one gets the sharper estimates $0.0377 \leq \varrho(K) \leq 0.2357$. 
As a general rule, the term $e^{-\langle\xi, M \xi\rangle}$ goes fast to 0 as $\|\xi\| \rightarrow \infty$. It is therefore reasonable to approximate the multiple integral appearing in (7) by using a truncated integral

$$
\tau(M, b)=\int_{[0, b]^{n}} e^{-\langle\xi, M \xi\rangle} d \xi,
$$

which in turn can be evaluated with the help of any numerical integration technique. For instance, one may consider the quadrature formula

$$
\tau_{N}(M, b)=\left[\sum_{k_{1}=1}^{N} \ldots \sum_{k_{n}=1}^{N} e^{-\left\langle\xi^{(k)}, M \xi^{(k)}\right\rangle}\right]\left(\frac{b}{N}\right)^{n}
$$

obtained with a regular partition of the integration box $[0, b]^{n}$, and with function evaluation at the center

$$
\xi^{(k)}=\xi^{\left(k_{1}, \ldots, k_{n}\right)}=\left(\left(k_{1}-\frac{1}{2}\right) \frac{b}{N}, \ldots,\left(k_{n}-\frac{1}{2}\right) \frac{b}{N}\right)
$$

of each sub-box

$$
V^{(k)}=V^{\left(k_{1}, \ldots, k_{n}\right)}=\prod_{i=1}^{n}\left[\left(k_{i}-1\right) \frac{b}{N}, k_{i} \frac{b}{N}\right] .
$$

The quality of the numerical approximation technique can be controlled with the help of the next proposition. As usual, the notation

$$
\operatorname{erf}[\cdot]=\frac{2}{\sqrt{\pi}} \int_{0}^{(\cdot)} e^{-t^{2}} d t
$$

stands for the Gaussian error function.

Proposition 3.5. Let $K$ be a polyhedral convex cone in $\mathbb{R}^{n}$ generated by $n$ linearly independent unit vectors. Let $M$ be the Gramian matrix associated to the generators. Then,

$$
\varrho(K)=2 \frac{\sqrt{\operatorname{det} M}}{\pi^{n / 2}}\left(\tau_{N}(M, b)+\varepsilon_{1}+\varepsilon_{2}\right) .
$$

Here $\varepsilon_{2}=\tau(M, b)-\tau_{N}(M, b)$ is the error induced by the quadrature formula (12) and

$$
\varepsilon_{1}=\int_{\mathbb{R}_{+}^{n} \backslash[0, b]^{n}} e^{-\langle\xi M \xi\rangle} d \xi
$$


is the error due to the truncation of the domain of integration in (7). One has

$$
\frac{1-\left[\operatorname{erf}\left(b \sqrt{\mu_{\max }(M)}\right)\right]^{n}}{\left[\mu_{\max }(M)\right]^{n / 2}} \leq \frac{2^{n}}{\pi^{n / 2}} \varepsilon_{1} \leq \frac{1-\left[\operatorname{erf}\left(b \sqrt{\mu_{\min }(M)}\right)\right]^{n}}{\left[\mu_{\min }(M)\right]^{n / 2}} .
$$

Proof. Formula (13) is a direct consequence of Lemma 3.1. On the other hand, it is clear that

$$
\int_{\mathbb{R}_{+}^{n} \backslash[0, b]^{n}} e^{-\mu_{\max }(M)\|\xi\|^{2}} d \xi \leq \varepsilon_{1} \leq \int_{\mathbb{R}_{+}^{n} \backslash[0, b]^{n}} e^{-\mu_{\min }(M)\|\xi\|^{2}} d \xi .
$$

But

$$
\begin{aligned}
\int_{\mathbb{R}_{+}^{n} \backslash[0, b]^{n}} e^{-a\|\xi\|^{2}} d \xi & =\int_{\mathbb{R}_{+}^{n}} e^{-a\|\xi\|^{2}} d \xi-\int_{[0, b]^{n}} e^{-a\|\xi\|^{2}} d \xi \\
& =\left[\int_{0}^{\infty} e^{-a u^{2}} d u\right]^{n}-\left[\int_{0}^{b} e^{-a u^{2}} d u\right]^{n} \\
& =\left[\frac{\pi}{4 a}\right]^{n / 2}\left\{1-[\operatorname{erf}(b \sqrt{a})]^{n}\right\}
\end{aligned}
$$

for any positive real $a$.

By proceeding in a standard way, one can obtain also an upper estimate for $\left|\varepsilon_{2}\right|$. Notice that

$$
\begin{aligned}
\left|\varepsilon_{2}\right| & =\left|\sum_{k_{1}=1}^{N} \ldots \sum_{k_{n}=1}^{N}\left\{\int_{V^{(k)}} e^{-\langle\xi, M \xi\rangle} d \xi-e^{-\left\langle\xi^{(k)}, M \xi^{(k)}\right\rangle}\left(\frac{b}{N}\right)^{n}\right\}\right| \\
& \leq \sum_{k_{1}=1}^{N} \ldots \sum_{k_{n}=1}^{N} \int_{V^{(k)}}\left|e^{-\langle\xi, M \xi\rangle}-e^{-\left\langle\xi^{(k)}, M \xi^{(k)}\right\rangle}\right| d \xi \\
& \leq\left[\sum_{k_{1}=1}^{N} \ldots \sum_{k_{n}=1}^{N} \gamma^{(k)}\right]\left(\frac{b}{N}\right)^{n}
\end{aligned}
$$

with

$$
\gamma^{(k)}=\sup _{\xi \in V^{(k)}}\left|e^{-\langle\xi, M \xi\rangle}-e^{-\left\langle\xi^{(k)}, M \xi^{(k)}\right\rangle}\right|
$$

bounding the quadrature error over the sub-box $V^{(k)}$. The above supremum could be worked out in detail, but it is not worthwhile spending too much effort on this point. 
We have tested the numerical integration method on the cone $K$ of Example 3.4. The same cone will be used later for testing other methods as well. The impact of the truncation level $b$ and the mesh size $b / N$ can be seen in Table 1 . The best estimate of $\varrho(K)$ is to be found in the right lower corner.

\begin{tabular}{|c|c|c|c|}
\hline$b / N$ & $b=3$ & $b=4$ & $b=5$ \\
\hline 0.10 & 0.080797 & 0.080810 & 0.080810 \\
\hline 0.05 & 0.080847 & 0.080861 & 0.080861 \\
\hline 0.02 & 0.080862 & 0.080875 & 0.080875 \\
\hline
\end{tabular}

Table 1 - Estimation of the volumetric modulus of the cone $K$ of Example 3.4 by using the numerical integration method.

It is important to choose the parameter $b$ in an appropriate manner. For the cone of Example 3.4, the truncation error $\varepsilon_{1}$ is sandwiched as follows:

$$
0.61685 \frac{1-[\operatorname{erf}(1.26798 b)]^{4}}{2.58492} \leq \varepsilon_{1} \leq 0.61685 \frac{1-[\operatorname{erf}(0.80178 b)]^{4}}{0.41327} .
$$

One sees that $b=4$ yields already a fairly small truncation error, namely, $\varepsilon_{1} \leq 3.4 \times 10^{-5}$.

\section{Multivariate power series method}

The $n$-dimensional version of Example 2.2 has been treated by Ribando [20]. This author proposes estimating the volumetric modulus of a polyhedral convex cone $K$ with the help of a multivariate power series $\sum_{r} a_{r} z^{r}$ in the $n(n-1) / 2$ variables

$$
z=\left(\left\langle g^{1}, g^{2}\right\rangle, \ldots,\left\langle g^{1}, g^{n}\right\rangle,\left\langle g^{2}, g^{3}\right\rangle, \ldots,\left\langle g^{n-1}, g^{n}\right\rangle\right) .
$$

The vector (14) collects the entries appearing in the upper triangular part of the Gramian matrix $M$. The multinomial notation $z^{r}$ has its usual meaning, i.e.,

$$
z^{r}=\left\langle g^{1}, g^{2}\right\rangle^{r_{1,2}} \ldots\left\langle g^{1}, g^{n}\right\rangle^{r_{1, n}}\left\langle g^{2}, g^{3}\right\rangle^{r_{2,3}} \ldots\left\langle g^{n-1}, g^{n}\right\rangle^{r_{n-1, n}} .
$$

The multi-index $r=\left(r_{1,2}, \ldots, r_{1, n}, r_{2,3}, \ldots, r_{n-1, n}\right)$ in the summation symbol $\sum_{r}$ runs over $\mathbb{N}^{n(n-1) / 2}$. The sum of all entries of $r$ is denoted by

$$
|r|=\sum_{i<j} r_{i, j}
$$


Given that $r$ is a vector, there is no risk of confusion with the absolute value notation. We also need the notation

$$
r_{j, i}=\left\{\begin{array}{lll}
1 & \text { if } & j=i \\
r_{i, j} & \text { if } & j>i .
\end{array}\right.
$$

Theorem 4.1 (cf. [20]). Let $K$ be a polyhedral convex cone in $\mathbb{R}^{n}$ generated by $n$ linearly independent unit vectors $\left\{g^{1}, \ldots, g^{n}\right\}$. Let $M$ be the Gramian matrix associated to the generators of $K$. Then,

(a) The volumetric modulus of $K$ is given by

$$
\varrho(K)=\left(\frac{1}{2}\right)^{n-1} \sqrt{\operatorname{det} M} \sum_{r} a_{r} z^{r}
$$

in case of convergence of the multivariate power series. Here, the coefficient $a_{r}$ is defined by

$$
a_{r}=\frac{1}{(\pi)^{n / 2}} \frac{(-2)^{|r|}}{\prod_{i<j}\left(r_{i, j} !\right)} \prod_{\ell=1}^{n} \Gamma\left(\frac{1}{2} \sum_{j=1}^{n} r_{\ell, j}\right) .
$$

(b) The convergence of the power series is guaranteed if the matrix $\widehat{M}$, given by

$$
\widehat{M}_{i, j}= \begin{cases}1 & \text { if } i=j, \\ -\left|\left\langle g^{i}, g^{j}\right\rangle\right| & \text { if } i \neq j,\end{cases}
$$

is positive definite.

As one can see, the coefficient $a_{r}$ is quite complicated. Besides, formula (15) is only valid on the domain of convergence of the power series. Anyway, one may consider evaluating the volumetric modulus of $K$ by using a truncated form of (15), namely,

$$
\varrho(K) \approx\left(\frac{1}{2}\right)^{n-1} \sqrt{\operatorname{det} M} \sum_{|r| \leq m} a_{r} z^{r} .
$$

We refer to (17) as the $m$-th order Ribando approximation of $\varrho(K)$. One can check that

$$
a_{r}=\left\{\begin{array}{lll}
1 & \text { if } & |r|=0, \\
-2 / \pi & \text { if } & |r|=1,
\end{array}\right.
$$


so the first-order Ribando approximation of $\varrho(K)$ takes the form

$$
\varrho(K) \approx\left(\frac{1}{2}\right)^{n-1} \sqrt{\operatorname{det} M}\left(1-\frac{2}{\pi} \sum_{i<j}\left\langle g^{i}, g^{j}\right\rangle\right) .
$$

Second and higher-order approximations must be worked out with the help of the computer.

We have evaluated the volumetric modulus of the cone $K$ of Example 3.4 by using (17). For this particular cone, the matrix (16) is given by

$$
\widehat{M}=\left[\begin{array}{cccc}
1 & -1 / 10 & -5 / 14 & -3 / 22 \\
-1 / 10 & 1 & -11 / 70 & -51 / 110 \\
-5 / 14 & -11 / 70 & 1 & -20 / 77 \\
-3 / 22 & -51 / 110 & -20 / 77 & 1
\end{array}\right] .
$$

Since $\lambda_{\min }(\widehat{M})=0.25256$ is positive, we are then in the region of validity of formula (15). Table 2 shows the quality of the estimation (17) as function of the truncation level $m$. As expected, the best results are obtained for large values of $m$. The cases $m=20$ and $m=40$ are undistinguishable because the associated estimates differ only after the sixth decimal place.

\begin{tabular}{|c|c|c|c|c|c|c|}
\hline$m=0$ & $m=1$ & $m=2$ & $m=5$ & $m=10$ & $m=20$ & $m=40$ \\
\hline 0.097403 & 0.067204 & 0.082871 & 0.079939 & 0.080930 & 0.080878 & 0.080878 \\
\hline
\end{tabular}

Table 2 - Estimation (17) of the volumetric modulus of the cone $K$ of Example 3.4.

Remark 4.2. Positive definiteness of the matrix $\widehat{M}$ is a fundamental assumption for the applicability of the power series method. For instance, if $K$ is generated by the vectors

$$
g^{1}=\left[\begin{array}{c}
\sqrt{2} / 2 \\
\sqrt{2} / 2 \\
0
\end{array}\right], g^{2}=\left[\begin{array}{c}
\sqrt{2} / 2 \\
0 \\
\sqrt{2} / 2
\end{array}\right], g^{3}=\left[\begin{array}{c}
\mu \\
\sqrt{\frac{1-\mu^{2}}{2}} \\
\sqrt{\frac{1-\mu^{2}}{2}}
\end{array}\right],
$$

then one observes the following two computational facts. Firstly, if one chooses $\mu=0.01$, then

$$
\lambda_{\min }(\widehat{M}) \approx-0.0094<0 .
$$


Numerical experimentation with this particular choice confirms the failure of convergence of the power series $\sum_{r} a_{r} z^{r}$. And, secondly, if one chooses $\mu=-0.01$, then

$$
\lambda_{\min }(\widehat{M}) \approx 0.095>0 .
$$

As predicted by the theory, the power series $\sum_{r} a_{r} z^{r}$ converges. However, it does it very slowly because $\widehat{M}$ is nearly singular.

Besides its use as tool for computing volumetric moduli, Theorem 4.1 has also some theoretical implications. By way of example, we state the following corollary.

Corollary 4.3. Let $K$ be a polyhedral convex cone in $\mathbb{R}^{n}$ generated by $n$ linearly independent unit vectors. Suppose that all pairs of generators form the same angle, say

$$
\psi \in] \arccos \left(\frac{1}{n-1}\right), \pi-\arccos \left(\frac{1}{n-1}\right)[.
$$

Then,

$$
\varrho(K)=\left(\frac{\sqrt{1-\cos \psi}}{2}\right)^{n-1} \sqrt{1+(n-1) \cos \psi} P(\cos \psi)
$$

where $P(x)=\sum_{q=0}^{\infty} c_{q} x^{q}$ is a real variable power series whose general term is given by

$$
c_{q}=\frac{(-2)^{q}}{(\pi)^{n / 2}} \sum_{|r|=q} \frac{1}{\prod_{i<j}\left(r_{i, j} !\right)} \prod_{\ell=1}^{n} \Gamma\left(\frac{1}{2} \sum_{j=1}^{n} r_{\ell, j}\right) .
$$

Proof. Let $M$ be the Gramian matrix associated to the generators $\left\{g^{1}, \ldots, g^{n}\right\}$ of the cone. By assumption, one has $\left\langle g^{i}, g^{j}\right\rangle=\cos \psi$ whenever $i \neq j$. As shown in [19, Lemma 3], this equi-angularity condition implies that

$$
\operatorname{det} M=(1-\cos \psi)^{n-1}[1+(n-1) \cos \psi] .
$$

One also has

$$
\sum_{r} a_{r} z^{r}=\sum_{q=0}^{\infty} \underbrace{\left[\sum_{|r|=q} a_{r}\right]}_{c_{q}}(\cos \psi)^{q} .
$$


By plugging all this information in (15), one arrives at the formula (20). On the other hand, $\sum_{q=0}^{\infty} c_{q} x^{q}$ converges for any $x \in \mathbb{R}$ such that the matrix $\widehat{M}$, given by

$$
\widehat{M}_{i, j}= \begin{cases}1 & \text { if } i=j, \\ -|x| & \text { if } i \neq j,\end{cases}
$$

is positive definite. In other words, the power series $P$ converges on $]-1 /(n-1)$, $1 /(n-1)[$. This explains why we are asking $\psi$ to satisfy the condition (19).

In view of (18), one has $c_{0}=1$ and $c_{1}=-n(n-1) / \pi$. Evaluating the coefficient $c_{2}$ is a more involved task. A matter of computation shows that

$$
a_{r}=\left\{\begin{array}{lll}
1 / 2 & \text { if } & |r|=2 \text { and Case I occurs, } \\
2 / \pi & \text { if } & |r|=2 \text { and Case II occurs, } \\
4 / \pi^{2} & \text { if } & |r|=2 \text { and Case III occurs, }
\end{array}\right.
$$

where Case I occurs when the multi-index $r$ contains a " 2 ", Case II refers to the configuration

$$
r_{i_{1}, j_{1}}=1, r_{i_{2}, j_{2}}=1 \text { with } i_{1}=i_{2} \text { or } j_{1}=j_{2},
$$

and Case III refers to the the last possible alternative, i.e.,

$$
r_{i_{1}, j_{1}}=1, r_{i_{2}, j_{2}}=1 \text { with } i_{1} \neq i_{2} \text { and } j_{1} \neq j_{2} \text {. }
$$

One gets in this way

$$
c_{2}=\frac{n(n-1)}{2}\left(\frac{1}{2}\right)+\chi_{n}\left(\frac{2}{\pi}\right)+\left[\frac{n(n-1)}{4}\left(\frac{n(n-1)}{2}-1\right)-\chi_{n}\right]\left(\frac{4}{\pi^{2}}\right),
$$

where

$$
\chi_{n}=\frac{n(n-1)(n-2)}{3}
$$

counts the number of ways of forming the configuration (21).

Remark 4.4. The condition (19) forces $\psi$ to be in a rather narrow interval around $\pi / 2$. For the particular choice $\psi=\pi / 2$, one obtains again the formula of Corollary 3.2. Suppose now that $\psi$ slightly deviates from orthogonality, i.e. $\psi=(\pi / 2)+\varepsilon$. In such a case,

$$
\varrho(K) \approx\left(\frac{1}{2}\right)^{n-1}\left[1+\frac{n(n-1)}{\pi} \varepsilon\right] .
$$


To see this, one needs to differentiate the right-hand side of (20) with respect to the variable $\psi$, and then one has to evaluate the derivative at $\pi / 2$. Of course, second-order differentiation of (20) leads to an approximation formula that incorporates an additional term in $\varepsilon^{2}$.

\section{Random techniques}

Let $\mathcal{L}(X)$ denotes the distribution law of a random vector $X$. An $n$-dimensional random vector $X$ has a spherically symmetric distribution if

$$
\mathcal{L}(X)=\mathcal{L}(Q X) \text { for all } Q \in \mathcal{O}_{n},
$$

where $\mathcal{O}_{n}$ stands for the group of orthogonal matrices of order $n$. Spherically symmetric distributions have been extensively studied in the literature, so we do not need to indulge in the analysis of these mathematical objects.

The next proposition is a key result of this section. The formulation of Proposition 5.1 is strikingly simple, but the consequences are manifold.

Proposition 5.1. Let $K \in \Xi\left(\mathbb{R}^{n}\right)$. Then,

$$
\varrho(K)=2 P[X \in K]
$$

for any absolutely continuous ${ }^{1} n$-dimensional random vector $X$ with spherically symmetric distribution.

Proof. Absolute continuity and spherical symmetry ensure that the random vector $X /\|X\|$ is well defined and uniformly distributed over $\mathbb{S}_{n}$ (cf. [5, Theorem 2.1]). Hence,

$$
P[X \in K]=P\left[\frac{X}{\|X\|} \in K \cap \mathbb{S}_{n}\right]=\frac{\operatorname{vol}_{n-1}\left(K \cap \mathbb{S}_{n}\right)}{\operatorname{vol}_{n-1}\left(\mathbb{S}_{n}\right)}=\frac{1}{2} \varrho(K) .
$$

For all practical purposes, think of $X$ as a Gaussian vector, i.e., normally distributed with the origin as mathematical expectation and with the identity matrix as covariance matrix. This is the most conspicuous example of an absolutely

\footnotetext{
${ }^{1}$ Absolute continuity is not strictly necessary in Proposition 5.1. The assumption $P[X=0]=$ 0 is all what is needed.
} 
continuous random vector satisfying the spherical symmetry requirement (22). Another useful option is considering $X$ as a random vector with uniform probability distribution over the unit sphere $\mathbb{S}_{n}$. The advantage of latter choice is that one does not have to worry about normalization since, by construction, the random vector is already normalized.

Despite its simplicity, Proposition 5.1 is a powerful tool for computing the volumetric modulus of a large variety of convex cones. As way of illustration, we mention the case of a specially structured polyhedral convex cone arising in maximum likelihood estimation (cf. $[4,11])$.

Corollary 5.2. The downward monotonic cone

$$
D_{n}=\left\{x \in \mathbb{R}^{n}: x_{1} \geq \ldots \geq x_{n}\right\}
$$

has a volumetric modulus equal to $2 / n$ !.

Proof. If $X$ is an $n$-dimensional vector, then formula (23) yields

$$
\begin{aligned}
\varrho\left(D_{n}\right) & =2 P\left[X_{1} \geq \ldots \geq X_{n}\right] \\
& =2 \int_{-\infty}^{\infty} \int_{-\infty}^{x_{1}} \cdots \int_{-\infty}^{x_{n-1}} \frac{1}{(2 \pi)^{n / 2}} e^{-\left(x_{1}^{2}+\ldots+x_{n}^{2}\right) / 2} d x_{n} \cdots d x_{2} d x_{1} .
\end{aligned}
$$

The above multiple integral can be evaluated by integrating first with respect to $x_{n}$, then with respect to $x_{n-1}$, and so on.

The upward monotonic cone

$$
U_{n}=\left\{x \in \mathbb{R}^{n}: x_{1} \leq \ldots \leq x_{n}\right\}
$$

has the same volumetric modulus as $D_{n}$. In general,

$$
\varrho\left(\left\{x \in \mathbb{R}^{n}: x_{\sigma(1)} \geq \ldots \geq x_{\sigma(n)}\right\}\right)=2 / n !
$$

for any permutation $\sigma$ on $\{1, \ldots, n\}$. This is a consequence of the fact that $\varrho: \Xi\left(\mathbb{R}^{n}\right) \rightarrow \mathbb{R}$ is orthogonally invariant.

In unimodal regression theory [2], a vector $x \in \mathbb{R}^{n}$ is called unimodal with a mode at the $q$-th component ( $q$-unimodal, for short) if

$$
x_{1} \leq \ldots \leq x_{q-1} \leq x_{q} \geq x_{q+1} \geq \ldots \geq x_{n} .
$$


For applications of the concept of unimodality in other areas of mathematics, see the interesting survey by Stanley [24]. Let $U_{q, n}$ denote the set of all $q$ unimodal vectors of $\mathbb{R}^{n}$. Clearly, $U_{1, n}$ corresponds to the downward monotonic cone $D_{n}$, and $U_{n, n}$ corresponds to the upward monotonic cone $U_{n}$. In general, the set $U_{q, n}$ is a polyhedral convex cone because it is expressible as intersection of $n-1$ half-spaces.

Proposition 5.3. Let $n \geq 3$. For any $q \in\{1, \ldots, n\}$, one has

$$
\varrho\left(U_{q, n}\right)=\frac{2}{n !}\left(\begin{array}{c}
n-1 \\
q-1
\end{array}\right)=\frac{2}{n(q-1) !(n-q) !} .
$$

In particular,

(a) $\varrho\left(U_{q, n}\right)=\varrho\left(U_{n-q+1, n}\right)$.

(b) $\varrho\left(U_{q, n}\right)$ is minimized at $q=1$ and at $q=n$.

(c) If $n$ is odd, then $\varrho\left(U_{q, n}\right)$ is maximized at $q=(n+1) / 2$.

(d) If $n$ is even, then $\varrho\left(U_{q, n}\right)$ is maximized at $q=n / 2$ and at $q=(n / 2)+1$.

Proof. Let $X$ be an $n$-dimensional Gaussian vector. Membership in $U_{q, n}$ is conditioned to membership in

$$
A_{q}=\left\{x \in \mathbb{R}^{n}: \max \left\{x_{1}, \ldots, x_{q-1}, x_{q+1}, \ldots, x_{n}\right\} \leq x_{q}\right\} .
$$

The fundamental law of conditional probabilities yields

$$
\begin{aligned}
P\left[X \in U_{q, n}\right]= & P\left[X \in U_{q, n} \mid X \in A_{q}\right] P\left[X \in A_{q}\right] \\
& +\underbrace{P\left[X \in U_{q, n} \mid X \notin A_{q}\right]}_{=0} P\left[X \notin A_{q}\right] .
\end{aligned}
$$

Clearly, $P\left[X \in A_{q}\right]=1 / n$. On the other hand, stochastic independence of the components of $X$ and Corollary 5.2 yield

$$
\begin{aligned}
P\left[X \in U_{q, n} \mid X \in A_{q}\right] & =P\left[X_{1} \leq \ldots \leq X_{q-1}, X_{q+1} \geq \ldots \geq X_{n}\right] \\
& =\underbrace{P\left[X_{1} \leq \ldots \leq X_{q-1}\right]}_{1 /(q-1) !} \underbrace{P\left[X_{q+1} \geq \ldots \geq X_{n}\right]}_{1 /(n-q) !} .
\end{aligned}
$$

Proposition 5.1 completes the proof of (24). The by-products (a)-(d) are immediate, see Table 3 for a quick overview. 


\begin{tabular}{|c|c|c|c|c|}
\hline$q$ & $n=3$ & $n=4$ & $n=5$ & $n=6$ \\
\hline 1 & $1 / 3$ & $1 / 12$ & $1 / 60$ & $1 / 360$ \\
\hline 2 & $2 / 3$ & $1 / 4$ & $1 / 15$ & $1 / 72$ \\
\hline 3 & $1 / 3$ & $1 / 4$ & $1 / 10$ & $1 / 36$ \\
\hline 4 & - & $1 / 12$ & $1 / 15$ & $1 / 36$ \\
\hline 5 & - & - & $1 / 60$ & $1 / 72$ \\
\hline 6 & - & - & - & $1 / 360$ \\
\hline
\end{tabular}

Table 3 - Volumetric moduli of the unimodal cones $U_{q, n}$.

Proposition 5.1 can also be used for estimating the volumetric modulus of a convex cone $K$ having no structure whatsoever. The basic idea is producing a large sample

$$
X^{(1)}, X^{(2)}, \ldots, X^{(N)} \equiv\left\{\begin{array}{l}
\text { stochastically independent } \\
n \text {-dimensional Gaussian vectors }
\end{array}\right.
$$

and counting the number of times that the target $K$ is being hit. If one introduces the Bernoulli variables

$$
Y_{i}=\left\{\begin{array}{lll}
1 & \text { if } & X^{(i)} \in K, \\
0 & \text { if } & \text { otherwise }
\end{array}\right.
$$

then Proposition 5.1 and the law of large numbers yield the approximation

$$
\varrho(K) \approx 2 \frac{Y_{1}+\ldots+Y_{N}}{N} .
$$

We have tested such a random approximation technique on the cone of Example 3.4. As shown in Table 4, the size of the random sample is a key factor for obtaining an acceptable degree of approximation. Confidence intervals at a $99 \%$ confidence level are also provided. Of course, sharper confidence intervals are obtained if one contents oneself with a confidence level at $95 \%$, as is common in practice.

Table 5 reports on the random estimation technique applied to the Lorentz cone $L_{n}$, the Pareto cone $\mathbb{R}_{+}^{n}$, and the downward monotonic cone $D_{n}$. The estimates for the Pareto cone are consistent with the values predicted by the formula $\varrho\left(\mathbb{R}_{+}^{n}\right)=(1 / 2)^{n-1}$. Consistency is also observed in the case of the downward monotonic cone whose volumetric modulus is given by Corollary 5.2 , and in the case of the Lorentz cone whose volumetric modulus is given by Corollary 2.4 . 


\begin{tabular}{|c|c|c|c|c|}
\hline Size of sample & $10^{7}$ & $10^{8}$ & $10^{9}$ & $10^{10}$ \\
\hline Estimation (25) & 0.080770 & 0.080851 & 0.080884 & 0.080874 \\
\hline Confidence interval & {$[0.080449$,} & {$[0.080750$,} & {$[0.080852$,} & {$[0.080864$,} \\
at the 99\% level & $0.081091]$ & $0.080952]$ & $0.080916]$ & $0.080884]$ \\
\hline
\end{tabular}

Table 4 - Random estimation technique for the volumetric modulus of the cone $K$ of Example 3.4.

\begin{tabular}{|c|c|c|c|}
\hline & $n=4$ & $n=5$ & $n=6$ \\
\hline$\varrho\left(L_{n}\right)$ & $\mathbf{0 . 1 8 1 6 9 0}$ & $\mathbf{0 . 1 1 6 1 1 7}$ & $\mathbf{0 . 0 7 5 5 8 7}$ \\
& 0.181684 & 0.116119 & 0.075587 \\
\hline$\varrho\left(\mathbb{R}_{+}^{n}\right)$ & $\mathbf{0 . 1 2 5 0 0 0}$ & $\mathbf{0 . 0 6 2 5 0 0}$ & $\mathbf{0 . 0 3 1 2 5 0}$ \\
& 0.125000 & 0.062500 & 0.031252 \\
\hline$\varrho\left(D_{n}\right)$ & $\mathbf{0 . 0 8 3 3 3 3}$ & $\mathbf{0 . 0 1 6 6 6 7}$ & $\mathbf{0 . 0 0 2 7 7 8}$ \\
& 0.083335 & 0.016665 & 0.002778 \\
\hline
\end{tabular}

Table 5 - Volumetric moduli of the Lorentz cone, the Pareto cone, and the downward monotonic cone. For each dimension $n$, one uses a sample of $10^{10}$ stochastically independent Gaussian vectors. Exact values are indicated in bold characters.

\section{Divide-and-conquer strategy}

Sometimes it helpful to decompose a convex cone $K$ as finite union

$$
K=K_{1} \cup \ldots \cup K_{\ell},
$$

and then compute the volumetric modulus of each component. Measure disjointness of the components can be ensured by assuming a suitable separation property. The rational behind such a divide-and-conquer method is explained in the next proposition.

Proposition 6.1. Let $K \in \Xi\left(\mathbb{R}^{n}\right)$ be decomposed as in (26), where $\left\{K_{1}, \ldots\right.$, $\left.K_{\ell}\right\}$ are elements of $\Xi\left(\mathbb{R}^{n}\right)$ satisfying the separation assumption

$$
\operatorname{dim}\left[\operatorname{span}\left(K_{i} \cap K_{j}\right)\right] \leq n-1 \quad \text { for all } i \neq j .
$$

Then, $\varrho(K)=\sum_{i=1}^{\ell} \varrho\left(K_{i}\right)$. 
Proof. By De Moivre inclusion-exclusion principle for the volume of a finite union, one has

$$
\begin{aligned}
\operatorname{vol}\left(K \cap \mathbb{B}_{n}\right) & =\operatorname{vol}\left(\bigcup_{i=1}^{\ell}\left(K_{i} \cap \mathbb{B}_{n}\right)\right) \\
& =\sum_{k=1}^{\ell}(-1)^{k-1} \sum_{\substack{I \subset\{1, \ldots, \ell\} \\
\operatorname{card}(I)=k}} \operatorname{vol}\left(\bigcap_{i \in I}\left(K_{i} \cap \mathbb{B}_{n}\right)\right) .
\end{aligned}
$$

The property (27) says that the linear space spanned by each intersection $K_{i} \cap K_{j}$ has dimension less than $n$. Hence, $\cap_{i \in I}\left(K_{i} \cap \mathbb{B}_{n}\right)$ has zero volume whenever $\operatorname{card}(I) \geq 2$. One gets in this way

$$
\operatorname{vol}\left(K \cap \mathbb{B}_{n}\right)=\sum_{i=1}^{\ell} \operatorname{vol}\left(K_{i} \cap \mathbb{B}_{n}\right),
$$

remaining now to divide on each side by the half-volume of $\mathbb{B}_{n}$.

Proposition 6.1 is fairly simple as a mathematical result. The two examples below illustrates how such proposition works in practice.

Example 6.2. In the same way as Archimedes approximates a circle by a $p$ sided polygon, one can approximate the three-dimensional Lorentz cone $L_{3}$ by a $p$-faced pyramidal cone

$$
\Lambda_{p}=\operatorname{cone}\left\{\gamma\left(t_{1}\right), \ldots, \gamma\left(t_{p}\right)\right\},
$$

where $\gamma(t)=(1 / \sqrt{2})(\cos t, \sin t, 1)^{T}$ and $t_{i}=(2 i-1) \pi / p$ for all $i \in$ $\{1, \ldots, p\}$. Suppose that $p \geq 4$. How to compute the volumetric modulus of $\Lambda_{p}$ ? In view of the linear dependence of the generators, neither the numerical integration method, nor the power series method can be applied in this case. A natural alternative is using a divide-and-conquer strategy: one decomposes (28) as a union of $p$ measure-disjoint pieces, namely, $K_{i}=\operatorname{cone}\left\{\gamma\left(t_{i}\right), \gamma\left(t_{i+1}\right), e_{3}\right\}$. Here $e_{3}=(0,0,1)^{T}$ and, by convention, $t_{p+1}=t_{1}$. A matter of symmetry shows that all the components $K_{i}$ have the same volumetric modulus. Hence, by applying Proposition 6.1 and the triple product formula (5), one gets

$$
\varrho\left(\Lambda_{p}\right)=p \varrho\left(K_{1}\right)=\frac{p}{\pi} \arctan \left[\frac{\sin (2 \pi / p)}{3+2 \sqrt{2}+\cos (2 \pi / p)}\right] .
$$


Note that $\lim _{p \rightarrow \infty} \varrho\left(\Lambda_{p}\right)=1-(\sqrt{2} / 2)=\varrho\left(L_{3}\right)$, in consistency with Proposition 2.1 and Corollary 2.4 .

Example 6.3. How to compute the volumetric modulus of a polyhedral convex cone

$$
K=\left\{x \in \mathbb{R}^{n}:\left\langle h^{j}, x\right\rangle \geq 0 \text { for all } j=1, \ldots, n-1\right\}
$$

given by a collection $\left\{h^{1}, \ldots, h^{n-1}\right\}$ of only $n-1$ linearly independents unit vectors of $\mathbb{R}^{n}$ ? Again, the numerical integration method and the power series method must be ruled out. We introduce then an additional vector $h^{n}$ whose role is cutting $K$ into two measure-disjoint pieces:

$$
\begin{aligned}
& K_{1}=\left\{x \in K:\left\langle h^{n}, x\right\rangle \geq 0\right\}, \\
& K_{2}=\left\{x \in K:\left\langle h^{n}, x\right\rangle \leq 0\right\} .
\end{aligned}
$$

The separation property (27) holds because $K_{1} \cap K_{2}$ is contained in the hyperplane with normal vector $h^{n}$. In the present situation, the cleverest way of defining the missing vector $h^{n}$ is by solving the linear system

$$
\left\langle h^{j}, h^{n}\right\rangle=0 \quad \text { for all } j \in\{1, \ldots, n-1\} .
$$

With such a choice of $h^{n}$, the components $K_{1}$ and $K_{2}$ have the same volumetric modulus. By Proposition 6.1, one has $\varrho(K)=2 \varrho\left(K_{1}\right)$. In order to evaluate $\varrho\left(K_{1}\right)$, one can use for instance the numerical integration method.

The purpose of Example 6.3 has been preparing the ground for handling a more general situation. The next proposition comes now without surprise.

Proposition 6.4. Consider a polyhedral convex cone

$$
K=\left\{x \in \mathbb{R}^{n}:\left\langle h^{j}, x\right\rangle \geq 0 \text { for all } j=1, \ldots, r\right\},
$$

where $r \leq n-1$ and $\left\{h^{1}, \ldots, h^{r}\right\}$ is a collection of linearly independent unit vectors of $\mathbb{R}^{n}$. Then,

$$
\varrho(K)=2^{n-r} \varrho(\widehat{K}),
$$

where $\widehat{K}$ is any simplicial reduction of $K$ in the sense that

$$
\widehat{K}=\left\{x \in \mathbb{R}^{n}:\left\langle h^{j}, x\right\rangle \geq 0 \text { for all } j=1, \ldots, n\right\}
$$


with $\left\{h^{r+1}, \ldots, h^{n}\right\}$ forming an orthonormal basis of the linear subspace $\left[\operatorname{span}\left\{h^{1}, \ldots, h^{r}\right\}\right]^{\perp}$.

Proof. If $\left\{h^{r+1}, \ldots, h^{n}\right\}$ forms a basis of the orthogonal complement of $\operatorname{span}\left\{h^{1}, \ldots, h^{r}\right\}$, then the full collection $\left\{h^{j}\right\}_{j=1}^{n}$ is linearly independent. Orthogonality of such basis ensures that the measure-disjoint pieces

$$
K_{i}=\left\{x \in K:\left\langle\varepsilon_{i, j} h^{j}, x\right\rangle \geq 0 \text { for all } j=r+1, \ldots, n\right\}
$$

have the same volumetric modulus. Each $K_{i}$ is associated to a vector $\left(\varepsilon_{i, r+1}, \ldots, \varepsilon_{i, n}\right)$ in the lattice $\{-1,1\}^{n-r}$. The convex cone $\widehat{K}$ is just one of the $2^{n-r}$ pieces listed in (31).

Recall that simplicial cone in $\mathbb{R}^{n}$ is a polyhedral convex cone generated by $n$ linearly independent vectors. This explains the use of the term "simplicial" while referring to $\widehat{K}$. The linearly independent generators $\left\{g^{1}, \ldots, g^{n}\right\}$ of $\widehat{K}$ are obtained by normalizing each column of the matrix $H\left(H^{T} H\right)^{-1}$, where $H=\left[h^{1}, \ldots, h^{n}\right]$. On the other hand, one uses the term "reduction" because $\widehat{K}$ is strictly contained in $K$.

Example 6.5. Let $V_{n}$ be the set of $n$-dimensional vectors whose secondorder differences are nonnegative, i.e.,

$$
V_{n}=\left\{x \in \mathbb{R}^{n}: x_{j+1}+x_{j-1}-2 x_{j} \geq 0 \text { for all } j=2, \ldots, n-1\right\} .
$$

The set $V_{n}$ is a polyhedral convex cone expressible as intersection of $n-2$ half-spaces. A simplicial reduction of $V_{n}$ can be constructed as follows. The normal vector corresponding to the $j$-th hyper-space is

$$
h^{j}=(\sqrt{6} / 6)(0, \ldots, 0,1,-2,1,0 \ldots, 0)^{T},
$$

where "-2" appears in the $j$-th coordinate. The scalar $\sqrt{6} / 6$ has been introduced just to make sure that $\left\|h^{j}\right\|=1$, although one could dispense with this normalization condition. The vectors

$$
\begin{aligned}
& u=(1,1, \ldots, 1)^{T}, \\
& v=(1-n, 3-n, 5-n, \ldots, n-3, n-1)^{T},
\end{aligned}
$$

are orthogonal among themselves, and also orthogonal to the vectors $\left\{h^{2}, \ldots\right.$, $\left.h^{n-1}\right\}$. So, one can take $h^{1}=u /\|u\|$ and $h^{n}=v /\|v\|$. 


\section{Comparing $\varrho$ with other solidity indices}

A solidity index in the axiomatic sense of [15] is a continuous function $g:\left(\Xi\left(\mathbb{R}^{n}\right), \delta\right) \rightarrow \mathbb{R}$ such that

- $g(K)=0$ if and only if $K$ is not solid,

- $g(K)=1$ if and only if $K$ is a half-space,

- $g$ is monotonic with respect to set inclusion,

- $g$ is invariant with respect to orthogonal transformations.

As mentioned in Section 2, the volumetric modulus $\varrho$ qualifies as a solidity index. Two other interesting examples of solidity indices are

$$
\varrho_{\text {met }}(K)=\inf _{\substack{Q \in \Xi\left(\mathbb{R}^{n}\right) \\ Q \text { not solid }}} \delta(Q, K)
$$

and

$$
\varrho_{\text {frob }}(K)=\left\{\begin{array}{l}
\text { radius of the largest ball centered } \\
\text { at a unit vector and contained in } K .
\end{array}\right.
$$

The "metric" solidity index (32) has been extensively studied in $[15,16,18]$. It has been established in [18, Corollary 2] that

$$
\varrho_{\text {met }}(K)=\cos \left[\frac{\theta_{\max }\left(K^{+}\right)}{2}\right],
$$

where $K^{+}$stands for the dual cone of $K$ and

$$
\theta_{\max }(P)=\max _{x, y \in P \cap \mathbb{S}_{n}} \arccos \langle x, y\rangle
$$

denotes the maximal angle of $P \in \Xi\left(\mathbb{R}^{n}\right)$. Concerning the "Frobenius" solidity index (33), a wide range of applications and relevant material can be found in $[6,8,9,13,14,15]$.

Example 7.1. Consider the case of a downward monotonic cone. It has been shown in [14] that

$$
\varrho_{\mathrm{frob}}\left(D_{n}\right)=\sqrt{\frac{6}{n(n-1)(n+1)}} \approx \frac{\sqrt{6}}{n^{3 / 2}} .
$$


On the other hand, by relying on (34) and [12, Proposition 2] one can prove that

$$
\varrho_{\text {met }}\left(D_{n}\right)=\cos \left[\left(1-\frac{1}{n}\right) \frac{\pi}{2}\right] \approx \frac{\pi}{2 n} .
$$

Note that $\varrho\left(D_{n}\right)=2 / n$ ! is "much" smaller than both (35) and (36).

Example 7.2. Let $a \in \mathbb{S}_{n}$. For a circular cone with half-aperture angle $\vartheta \in$ ] $0, \pi / 2[$ one obtains

$$
\varrho_{\text {frob }}\left(R_{a, \vartheta}\right)=\varrho_{\text {met }}\left(R_{a, \vartheta}\right)=\sin \vartheta
$$

a number which is independent of $n$. By constrast, $\varrho\left(R_{a, \vartheta}\right)$ does depend on $n$ as one can see from Proposition 2.3.

Examples 7.1 and 7.2 will do by way of illustration. The question that we would like to explore now is whether there is some kind of general relationship between $\varrho$ and the other two solidity indices. Observe that

$$
\varrho_{\text {frob }}(K) \leq \varrho_{\text {met }}(K) \text { for all } K \in \Xi\left(\mathbb{R}^{n}\right) \text {. }
$$

This is simply because $\varrho_{\text {met }}$ is the largest one among all the solidity indices that are nonexpansive (cf. [15]). The next definition will be useful.

Definition 7.3. Two solidity indices $g_{1}, g_{2}: \Xi\left(\mathbb{R}^{n}\right) \rightarrow \mathbb{R}^{n}$ are:

i) linearly comparable if there are positive constants $a$ and $b$, depending possibly on $n$, such that $a g_{2} \leq g_{1} \leq b g_{2}$.

ii) equivalent if there is an increasing surjection $\varphi:[0,1] \rightarrow[0,1]$ such that $g_{2}=\varphi \circ g_{1}$.

We start by stating two negative results ${ }^{2}$.

Proposition 7.4. $\varrho$ is neither linearly comparable to $\varrho_{\text {frob }}$ nor to $\varrho_{\text {met }}$.

\footnotetext{
${ }^{2}$ Note added in proof: We have been able, however, to prove that $\varrho \leq \varrho$ met. This result will be presented in a forthcoming publication.
} 
Proof. We prove the following claim: there is no positive constant $a$ such that $a \varrho_{\text {frob }} \leq \varrho$. To do this, we show that

$$
\inf _{\substack{K \in \Xi\left(\mathbb{R}^{n}\right) \\ K \text { solid }}} \frac{\varrho(K)}{\varrho_{\text {frob }}(K)}=0 .
$$

Let us evaluate the quotient $\varrho / \varrho_{\text {frob }}$ on a circular cone $R_{a, \vartheta}$ and then let $\vartheta$ go to zero. By applying L'Hôpital's rule, one gets

$$
\lim _{\vartheta \rightarrow 0^{+}} \frac{\varrho\left(R_{a, \vartheta}\right)}{\varrho_{\text {frob }}\left(R_{a, \vartheta}\right)}=\lim _{\vartheta \rightarrow 0^{+}} \frac{F_{n}(\vartheta)}{\sin \vartheta}=\lim _{\vartheta \rightarrow 0^{+}} \frac{\kappa_{n}^{-1}(\sin \vartheta)^{n-2}}{\cos \vartheta}=0 .
$$

The case of $\varrho_{\text {met }}$ is treated in exactly the same way.

Proposition 7.5. $\varrho$ is neither equivalent to $\varrho_{\text {frob }}$ nor to $\varrho_{\text {met }}$.

Proof. The function $F_{n}$ introduced in Proposition 2.3 is a bijection from $[0, \pi / 2]$ to $[0,1]$. Let $\vartheta_{n}$ be the unique solution to the nonlinear equation $F_{n}(\vartheta)=(1 / 2)^{n-1}$. Then $\varrho\left(R_{a, \vartheta_{n}}\right)=\varrho\left(\mathbb{R}_{+}^{n}\right)$, regardless of the choice of $a \in \mathbb{S}_{n}$. But, on the other hand,

$$
\varrho_{\mathrm{met}}\left(R_{a, \vartheta_{n}}\right)=\sin \vartheta_{n} \neq \sqrt{1 / 2}=\varrho_{\mathrm{met}}\left(\mathbb{R}_{+}^{n}\right) .
$$

This rules out the possibility of finding an increasing surjective function $\varphi$ : $[0,1] \rightarrow[0,1]$ such that $\varrho=\varphi \circ \varrho_{\text {met }}$. In short, $\varrho$ and $\varrho_{\text {met }}$ are not equivalent. One can also check that

$$
\varrho_{\text {frob }}\left(R_{a, \vartheta_{n}}\right)=\sin \vartheta_{n} \neq \sqrt{1 / n}=\varrho_{\text {frob }}\left(\mathbb{R}_{+}^{n}\right) .
$$

Hence, $\varrho$ is not equivalent to $\varrho_{\text {frob }}$ either.

Despite the negative results stated in Propositions 7.4 and 7.5, there is a link between $\varrho$ and $\varrho_{\text {frob }}$ after all. However, such a link is nonlinear in nature and quite sophisticated.

Theorem 7.6. For all $K \in \Xi\left(\mathbb{R}^{n}\right)$ one has

$$
\frac{1}{\kappa_{n}} \int_{0}^{\varrho_{\text {frob }}(K)} \frac{t^{n-2}}{\sqrt{1-t^{2}}} d t \leq \varrho(K),
$$


as well as

$$
1-\frac{1}{\kappa_{n}} \int_{0}^{\varrho \text { frob }\left(K^{+}\right)}\left[\sqrt{1-t^{2}}\right]^{n-3} d t \geq \varrho(K) .
$$

Both inequalities become equalities if and only if $K$ is a circular cone.

Proof. A natural idea that comes to mind is estimating $\varrho(K)$ by using the inner and outer circular approximations

$$
\begin{aligned}
& K_{\text {inn }} \equiv \text { largest circular cone contained in } K, \\
& K_{\text {out }} \equiv \text { smallest circular cone containing } K .
\end{aligned}
$$

Let $\theta_{\text {inn }}(K)$ and $\theta_{\text {out }}(K)$ denote the half-aperture angles of $K_{\text {inn }}$ and $K_{\text {out }}$, respectively. As a consequence of Proposition 2.3, one gets

$$
\frac{1}{\kappa_{n}} \int_{0}^{\theta_{\text {inn }}(K)}(\sin t)^{n-2} d t \leq \rho(K) \leq \frac{1}{\kappa_{n}} \int_{0}^{\theta_{\text {out }}(K)}(\sin t)^{n-2} d t .
$$

Let us work out both sides of the above sandwich. It can be shown that

$$
\begin{aligned}
\sin \left[\theta_{\text {inn }}(K)\right] & =\varrho_{\text {frob }}(K), \\
\cos \left[\theta_{\text {out }}(K)\right] & =\varrho_{\text {frob }}\left(K^{+}\right) .
\end{aligned}
$$

Formula (41) is obtained by combining [17, Theorem 7] and [15, Proposition 6.3]. Formula (40) is obtained from (41) by using duality arguments. Hence,

$$
\begin{aligned}
& \frac{1}{\kappa_{n}} \int_{0}^{\theta_{\text {inn }}(K)}(\sin t)^{n-2} d t=G_{n}\left(\varrho_{\text {frob }}(K)\right), \\
& \frac{1}{\kappa_{n}} \int_{0}^{\theta_{\text {out }}(K)}(\sin t)^{n-2} d t=H_{n}\left(\varrho_{\text {frob }}\left(K^{+}\right)\right)
\end{aligned}
$$

with $G_{n}, H_{n}:[0,1] \rightarrow[0,1]$ given respectively by

$$
\begin{aligned}
& G_{n}(r)=F_{n}(\arcsin r)=\frac{1}{\kappa_{n}} \int_{0}^{r} \frac{t^{n-2}}{\sqrt{1-t^{2}}} d t, \\
& H_{n}(r)=F_{n}(\arccos r)=1-\frac{1}{\kappa_{n}} \int_{0}^{r}\left[\sqrt{1-t^{2}}\right]^{n-3} d t .
\end{aligned}
$$

Finally, note that both inequalities in (39) become equalities if and only if $\theta_{\text {inn }}(K)=\theta_{\text {out }}(K)$. This is yet equivalent to saying that $K$ is a circular cone. 
Corollary 7.7. For all $K \in \Xi\left(\mathbb{R}^{n}\right)$ one has

$$
\frac{1}{(n-1) \kappa_{n}}\left[\varrho_{\text {frob }}(K)\right]^{n-1} \leq \varrho(K) .
$$

Proof. It is enough to observe that

$$
\frac{t^{n-2}}{\sqrt{1-t^{2}}} \geq t^{n-2}
$$

Thus, the inequality (42) is a weakening of (37).

We stated Corollary 7.7 just to indicate that $\varrho$ can be minorized by a positive multiple of the power $\left[\varrho_{\text {frob }}(\cdot)\right]^{n-1}$. Recall that $\varrho$ cannot be minorized by a positive multiple of $\varrho_{\text {frob }}$ itself.

\section{By way of conclusion}

In this work we have discussed several methods for computing the volumetric modulus of a convex cone. Table 6 gives a general overview of the advantages and disadvantages of each method. The details can be consulted in the corresponding section.

\begin{tabular}{|c|c|c|}
\hline & Advantage & Disadvantage \\
\hline $\begin{array}{c}\text { Numerical } \\
\text { integration }\end{array}$ & $\begin{array}{c}\text { Availability of error } \\
\text { estimates }\end{array}$ & $\begin{array}{c}\text { High computational cost, } \\
\text { Applies only to simplicial cones }\end{array}$ \\
\hline $\begin{array}{c}\text { Power } \\
\text { series }\end{array}$ & $\begin{array}{c}\text { Low computational } \\
\text { cost }\end{array}$ & $\begin{array}{c}\text { Must satisfy convergence test, } \\
\text { Applies only to simplicial cones }\end{array}$ \\
\hline $\begin{array}{c}\text { Random } \\
\text { technique }\end{array}$ & $\begin{array}{c}\text { Applies beyond the class } \\
\text { of polyhedral cones }\end{array}$ & $\begin{array}{c}\text { Random sample } \\
\text { must be huge }\end{array}$ \\
\hline $\begin{array}{c}\text { Divide } \\
\text {-and- } \\
\text { conquer }\end{array}$ & $\begin{array}{c}\text { Applies beyond } \\
\text { the class of } \\
\text { polyhedral cones }\end{array}$ & $\begin{array}{c}\text { Requires identifying } \\
\text { a measure-disjoint }\end{array}$ \\
\hline
\end{tabular}

Table 6 - Methods for computing volumetric moduli.

So, one has several computational methods at hand, and one can combine them to produce additional options. Despite this fact, there are important convex cones arising in practice for which none of the above methods is applicable. 
The next example concerns a nasty convex cone arising in Moving Average Estimation (cf. [1, 3]).

Example 8.1. Let $d \geq 2$. One says that $x=\left(x_{0}, \ldots, x_{d}\right) \in \mathbb{R}^{d+1}$ is an autocorrelation vector if there exists $z=\left(z_{0}, \ldots, z_{d}\right) \in \mathbb{R}^{d+1}$ such that

$$
x_{k}=\sum_{i=0}^{d-k} z_{i} z_{i+k} \text { for all } k \in\{0,1, \ldots, d\} .
$$

Let $C_{d+1}$ denote the set of all autocorrelation vectors in $\mathbb{R}^{d+1}$. It is known that $C_{d+1}$ is representable in the form

$$
C_{d+1}=\left\{x \in \mathbb{R}^{d+1}: x_{0}+2 \sum_{k=1}^{d} x_{k} \cos (k w) \geq 0, \forall w \in[0, \pi]\right\} .
$$

By using this frequency-domain characterization, it is not difficult to show that $C_{d+1}$ is a solid pointed closed convex cone.

The convex cone of Example 8.1 is not polyhedral. Hence, the numerical integration method and the power series method must be ruled out. On the other hand, it is not clear how to partition $C_{d+1}$ in terms of measure-disjoint convex cones with easily computable volumetric moduli. We are left with the random technique as only option. However, checking if a given vector belongs to $C_{d+1}$ is not a trivial matter, and the cost of this operation must be multiplied by the size of the random sample. Note that the right-hand side of (43) is a set defined by infinitely many contraints.

For dealing with a desperate situation like this, there are at least two possibilities. The first option is estimating $\varrho(K)$ by using the sandwich (39). In fact, one does not need actually to compute the exact values of $\theta_{\text {inn }}(K)$ and $\theta_{\text {out }}(K)$. Given the monotonicity of $\vartheta \mapsto \int_{0}^{\vartheta}(\sin t)^{n-2} d t$, it is perfectly acceptable to use a lower bound for $\theta_{\text {inn }}(K)$ and a upper bound for $\theta_{\text {out }}(K)$. Let us see how this principle works in the case of the cone of autocorrelation vectors.

Corollary 8.2. For $d \geq 2$, one has

$$
\frac{1}{\kappa_{d+1}} \int_{0}^{\alpha_{d}}(\sin t)^{d-1} d t \leq \varrho\left(C_{d+1}\right) \leq \frac{1}{\kappa_{d+1}} \int_{0}^{\beta_{d}}(\sin t)^{d-1} d t
$$


with

$$
\begin{aligned}
& \alpha_{d}=\arcsin [1 / \sqrt{1+4 d}] \\
& \beta_{d}=\arccos \left[\inf \left\{x_{0}: x \in C_{d+1},\|x\|=1\right\}\right] .
\end{aligned}
$$

Proof. Write $x=\left(x_{0}, \xi\right)$ with $x_{0} \in \mathbb{R}$ and $\xi \in \mathbb{R}^{d}$. For all $w \in[0, \pi]$, one has

$$
\begin{aligned}
x_{0}+2 \sum_{k=1}^{d} x_{k} \cos (k w) & \geq x_{0}-2\|\xi\|\left[\sum_{k=1}^{d} \cos ^{2}(k w)\right]^{1 / 2} \\
& \geq x_{0}-2\|\xi\| \sqrt{d} .
\end{aligned}
$$

By using these inequalities, one can show that the circular cone with axis $a=$ $(1,0, \ldots, 0) \in \mathbb{S}_{d+1}$ and half-aperture angle $\alpha_{d}$ is contained in $C_{d+1}$. Hence, $\alpha_{d}$ is a lower estimate for $\theta_{\mathrm{inn}}\left(C_{d+1}\right)$. On the other hand,

$$
\begin{aligned}
\cos \left[\theta_{\text {out }}\left(C_{d+1}\right)\right] & =\sup _{\|y\|=1} \inf _{\substack{x \in C_{d+1} \\
\|x\|=1}}\langle y, x\rangle \\
& \geq \inf \left\{x_{0}: x \in C_{d+1},\|x\|=1\right\}
\end{aligned}
$$

i.e., $\beta_{d}$ is a upper estimate for $\theta_{\text {out }}\left(C_{d+1}\right)$.

Table 7 displays the numerical values of the bounds (44) for the cases $d=2$, $d=3$, and $d=4$. The bounds for $\varrho\left(C_{d+1}\right)$ could be sharpened by using the exact values of $\theta_{\text {inn }}\left(C_{d+1}\right)$ and $\theta_{\text {out }}\left(C_{d+1}\right)$. However, one should not be over optimistic because $C_{d+1}$ is far from being a circular cone. The situation gets even worse when $d$ increases. We must say things as they are: the estimates given in Table 7 are very disappointing. The method of inner and outer approximation by circular cones is ill suited in the case of the cone of autocorrelation vectors.

\begin{tabular}{|c|c|c|c|c|}
\hline$d$ & $\alpha_{d}$ & $\beta_{d}$ & lower bound for $\varrho\left(C_{d+1}\right)$ & upper bound for $\varrho\left(C_{d+1}\right)$ \\
\hline 2 & 0.3398 & 0.6473 & 0.0572 & 0.2023 \\
\hline 3 & 0.2810 & 0.7603 & 0.0093 & 0.1661 \\
\hline 4 & 0.2450 & 0.8394 & 0.0013 & 0.1471 \\
\hline
\end{tabular}

Table 7 - Bounding $\varrho\left(C_{d+1}\right)$ by using inner and outer circular approximations. Figures are rounded to four decimals. 
A second and much better possibility for consideration is using an outer polyhedral approximation

$$
C_{d+1}^{\Omega}=\left\{x \in \mathbb{R}^{d+1}: x_{0}+2 \sum_{k=1}^{d} x_{k} \cos (k w) \geq 0, \forall w \in \Omega\right\}
$$

of the cone $C_{d+1}$. Here $\Omega=\left\{w_{0}, \ldots, w_{N}\right\}$ stands for a finite collection of points in $[0, \pi]$. One gets in this way the upper estimate

$$
\varrho\left(C_{d+1}\right) \leq \varrho\left(C_{d+1}^{\Omega}\right)
$$

In Table 8 one considers a regular partition of $[0, \pi]$, i.e., $w_{i}=i \pi / N$ for all $i \in\{0,1, \ldots, N\}$. This implies that (45) is a polyhedral convex cone defined by $\operatorname{card}(\Omega)=N+1$ contraints. The volumetric modulus of $C_{d+1}^{\Omega}$ is estimated by using the random technique. For obtaining each entry in Table 8 , one works with a sample of $10^{8}$ stochastically independent Gaussian vectors.

\begin{tabular}{|l|c|c|c|c|}
\hline$d$ & $N=10$ & $N=20$ & $N=50$ & $N=100$ \\
\hline 2 & 0.1091 & 0.1083 & 0.1080 & 0.1079 \\
\hline 3 & 0.0387 & 0.0359 & 0.0357 & 0.0356 \\
\hline 4 & 0.0137 & 0.0129 & 0.0126 & 0.0126 \\
\hline
\end{tabular}

Table 8 - Volumetric modulus of $C_{d+1}^{\Omega}$. Figures are rounded to four decimals.

As far as the first four decimals are concerned, the term $\varrho\left(C_{d+1}^{\Omega}\right)$ does not change significatively if the mesh parameter $N$ goes beyond 100 . Observe that the upper bounds for $\varrho\left(C_{d+1}\right)$ provided by the last column of Table 8 are much sharper than the corresponding upper bounds given in Table 7.

Remark 8.3. If $\Omega$ is a regular mesh whose cardinality goes to $\infty$, then $C_{d+1}^{\Omega}$ converges to $C_{d+1}$ with respect to the metric $\delta$. The proof of this fact is long and tedious, so it will not be presented here. Such a convergence result indicates that $\varrho\left(C_{d+1}^{\Omega}\right)$ can be made arbitrarily close to $\varrho\left(C_{d+1}\right)$ by suitably refining the mesh $\Omega$. 


\section{REFERENCES}

[1] B. Alkire and L. Vandenberghe, Convex optimization problems involving finite autocorrelation sequences. Math. Program., 93 (2002), 331-359.

[2] V. Boyarshinov and M. Magdon-Ismail, Linear time isotonic and unimodal regression in the $L^{1}$ and $L^{\infty}$ norms. J. Discrete Algorithms, 4 (2006), 676-691.

[3] B. Dumitrescu, P. Stoica and I. Tăbuş, On the parameterization of positive real sequences and MA parameter estimation. IEEE Trans. Signal Process., 49 (2001), 2630-2639.

[4] R.L. Dykstra and J.H. Lemke, Duality of I projections and maximum likelihood estimation for log-linear models under cone constraints. J. Amer. Statist. Assoc., 83 (1988), 546-554.

[5] M.L. Eaton and T. Kariya, Robust tests for spherical symmetry. Ann. Statist., 5 (1977), 206-215.

[6] M. Epelman and R.M. Freund, A new condition measure, preconditioners, and relations between different measures of conditioning for conic linear systems. SIAM J. Optim., 12 (2002), 627-655.

[7] F. Eriksson, On the measure of solid angles. Math. Mag., 63 (1990), 184-187.

[8] R.M. Freund, On the primal-dual geometry of level sets in linear and conic optimization. SIAM J. Optim., 13 (2003), 1004-1013.

[9] R.M. Freund and J.R. Vera, Condition-based complexity of convex optimization in conic linear form via the ellipsoid algorithm. SIAM J. Optim., 10 (1999), 155-176.

[10] M.P.C. Fossorier and A. Valembois, Sphere-packing bounds revisited for moderate block lengths. IEEE Trans. Inform. Theory, 50 (2004), 2998-3014.

[11] W. Gao and N.Z. Shi, I-projection onto isotonic cones and its applications to maximum likelihood estimation for log-linear models. Ann. Inst. Statist. Math., 55 (2003), 251-263.

[12] D. Gourion and A. Seeger, Critical angles in polyhedral convex cones: numerical and statistical considerations. Math. Program., 123 (2010), 173-198.

[13] R. Henrion and A. Seeger, On properties of different notions of centers for convex cone. Set-Valued Anal., 18 (2010), 205-231.

[14] R. Henrion and A. Seeger, Inradius and circumradius of various convex cones arising in applications. Set-Valued Anal., to appear.

[15] A. Iusem and A. Seeger, Axiomatization of the index of pointedness for closed convex cones. Comput. Applied Math., 24 (2005), 245-283.

[16] A. Iusem and A. Seeger, Measuring the degree of pointedness of a closed convex cone: a metric approach. Math. Nachr., 279 (2006), 599-618.

[17] A. Iusem and A. Seeger, Normality and modulability indices. Part I: convex cones in normed spaces. J. Math. Anal. Appl., 338 (2008), 392-406. 
[18] A. Iusem and A. Seeger, Antipodality in convex cones and distance to unpointedness. Appl. Math. Letters, 21 (2008), 1018-1023.

[19] A. Iusem and A. Seeger, Antipodal pairs, critical pairs, and Nash angular equilibria in convex cones. Optimiz. Meth. Software, 23 (2008), 73-93.

[20] J.M. Ribando, Measuring solid angles beyond dimension three. Discrete Comput. Geom., 36 (2006), 479-487.

[21] A. van Oosterom and J. Strackee, The solid angle of a plane triangle. IEEE Trans. Biomed. Eng., 30 (1983), 125-126.

[22] A. Seeger and M. Torki, Local minima of quadratic forms on convex cones. J. Global Optim., 44 (2009), 1-28.

[23] C. Shannon, Probability of error for optimal codes in a Gaussian channel. Bell System Tech. J., 38 (1959), 611-656.

[24] R.P. Stanley, Log-concave and unimodal sequences in algebra, combinatorics, and geometry. Graph theory and its applications: East and West (Jinan, 1986), Ann. New York Acad. Sci., 576 (1989), 500-535. 